Article

\title{
Polymeric Micelles Based on Modified Glycol Chitosan for Paclitaxel Delivery: Preparation, Characterization and Evaluation
}

\author{
Na Liang ${ }^{1}$, Shaoping Sun ${ }^{2, *}$, Xianfeng Gong ${ }^{2}$, Qiang $\mathrm{Li}^{2}$, Pengfei Yan ${ }^{2,3, *}$ and Fude Cui ${ }^{4}$ \\ 1 Key Laboratory of Photochemical Biomaterials and Energy Storage Materials, Heilongjiang Province, \\ College of Chemistry \& Chemical Engineering, Harbin Normal University, Harbin 150025, China; \\ liangna528@163.com \\ 2 Key Laboratory of Chemical Engineering Process \& Technology for High-Efficiency Conversion, College of \\ Heilongjiang Province; School of Chemistry and Material Science, Heilongjiang University, Harbin 150080, \\ China; gongxianfeng@sina.com (X.G.); liqianghlj100@163.com (Q.L.) \\ 3 Key Laboratory of Functional Inorganic Material Chemistry, Heilongjiang University, Harbin 150080, China \\ 4 School of Pharmacy, Shenyang Pharmaceutical University, Shenyang 110016, China; syphucuifude@163.com \\ * Correspondence: sunshaoping111@163.com (S.S.); yanpf@vip.sina.com (P.Y.); \\ Tel./Fax: +86-451-8660-8616 (S.S.)
}

Received: 22 April 2018; Accepted: 15 May 2018; Published: 23 May 2018

\begin{abstract}
Amphiphilic polymer of $\alpha$-tocopherol succinate modified glycol chitosan (TS-GC) was successfully constructed by conjugating $\alpha$-tocopherol succinate to the skeleton of glycol chitosan and characterized by Fourier-transform infrared (FT-IR) and proton nuclear magnetic resonance $\left({ }^{1} \mathrm{H}-\mathrm{NMR}\right)$. In aqueous milieu, the conjugates self-assembled to micelles with the critical aggregation concentration of $7.2 \times 10^{-3} \mathrm{mg} / \mathrm{mL}$. Transmission electron microscope (TEM) observation and dynamic light scattering (DLS) measurements were carried out to determine the physicochemical properties of the micelles. The results revealed that paclitaxel (PTX)-loaded TS-GC micelles were spherical in shape. Moreover, the PTX-loaded micelles showed increased particle sizes $(35 \mathrm{~nm}$ vs. $142 \mathrm{~nm})$ and a little reduced zeta potential $(+19 \mathrm{mV}$ vs. $+16 \mathrm{mV})$ compared with blank micelles. The X-ray diffraction (XRD) spectra demonstrated that PTX existed inside the micelles in amorphous or molecular state. In vitro and in vivo tests showed that the PTX-loaded TS-GC micelles had advantages over the Cremophor EL-based formulation in terms of low toxicity level and increased dose, which suggested the potential of the polymer as carriers for PTX to improve their delivery properties.
\end{abstract}

Keywords: glycol chitosan; $\alpha$-tocopherol succinate; amphiphilic polymer; micelles; paclitaxel

\section{Introduction}

Paclitaxel (PTX), as a powerful anti-tumor drug, has been extensively used in the clinical treatment of several solid tumors, such as refractory ovarian cancer, metastasis breast cancer, non-small cell lung cancer, Acquired Immune Deficiency Syndrome-related Kaposi's sarcoma and other cancers $[1,2]$. Due to its poor water solubility of approximately $<2 \mu \mathrm{g} / \mathrm{mL}$, PTX is currently solubilized in a 50:50 mixture of Cremophor EL (PEG-35 caster oil) and dehydrated ethanol as Taxol ${ }^{\circledR}$ (Bristol-Myers Squibb, New York City, NY, USA). However, several studies reported that Cremophor EL induced serious side effects such as hypersensitivity, nephrotoxicity, neurotoxicity, and the extraction of plasticizer from the infusion tubes [3]. In light of these drawbacks, a number of alternative preparations were investigated, including liposomes, nanocrystals, micelles, cyclodextrin complexes and PTX conjugates [4-8]. 
Among these formulations, polymeric micelles have been proven as promising drug delivery systems for PTX administration $[9,10]$, because of their attractive characteristics, such as biocompatibility, high drug-loading content, small size $(<200 \mathrm{~nm})$ and propensity to evade scavenging by the mononuclear phagocyte system (MPS) [11,12]. Moreover, the nanoscale dimensions of polymeric micelles permit their selectively accumulation in tumor tissues due to the enhanced permeability and retention (EPR) effect, which is termed "passive targeting" [13,14]. The formation of polymeric micelles is generally considered as the self-assembly of polymeric amphiphiles. In aqueous medium, polymeric amphiphiles form the micelles consisting of the inner hydrophobic core and the outer hydrophilic shell. The hydrophobic core provides a storeroom for loading hydrophobic drugs, and the hydrophilic shell allows retaining the stability of micelles in an aqueous environment [15].

Due to its favorable properties, such as biodegradability, biocompatibility, nontoxicity and bioadhesivity, chitosan has been widely studied as a pharmaceutical carrier for drug delivery [16,17]. However, chitosan is insoluble at $\mathrm{pH}$ values above its $\mathrm{pKa}(6.4)$ in water, and this obviously limits its biomedical applications.

In recent years, glycol chitosan (GC), which possesses good solubility over a broad range of $\mathrm{pH}$, has been studied to construct drug delivery systems for PTX, such as nanocrystals, nanoparticles, hydrogels and microspheres [18-21]. The hydrophobically modified GC, which could be used as micellar carriers, has been extensively studied [22,23].

$\alpha$-Tocopherol is a good solvent for many hydrophobic drugs because of its excellent lipophilic nature [24]. Once it is grafted on to the backbone of GC, it may serve as the hydrophobic segment and therefore provide sufficient capacity for poorly soluble drugs.

Inspired by the above investigations, in this study, an amphiphilic polymer $\alpha$-tocopherol succinate modified glycol chitosan (TS-GC) was designed for PTX delivery. The TS-GC was prepared through amide formation. The preparation, characterization and self-assembling ability of TS-GC were studied. Furthermore, the physicochemical properties, hemolysis, in vitro cytotoxicity and in vivo antitumor activity of PTX-loaded micelles were evaluated deeply.

\section{Results and Discussion}

\subsection{Synthesis and Characterization of TS-GC}

In this study, the polymer TS-GC was synthesized via the coupling reaction between carboxyl group of TS and amine group of GC in the presence of water-soluble 1-Ethyl-3-(3-dimethylaminopropyl) carbodiimide hydrochloride (EDC). Firstly, an active ester intermediate was formed between the carboxyl group of TS and EDC. Then the amino bond can easily formed by reaction of primary amino of GC with the intermediate. NHS was used to stabilize the intermediate to achieve higher yield [25]. The scheme of the reaction between GC and TS was shown in Figure 1.

${ }^{1} \mathrm{H}-\mathrm{NMR}$ spectra confirmed the grafting of TS chain onto GC as illustrated in Figure 2. In the spectrum of TS-GC, the proton peaks of TS, including methyl (0.77-0.88 ppm) and methylene (0.99-1.54 ppm) that belonged to the protons of the long-chain alkyl group of TS were observed. Moreover, the new-emerged signals at $2.45-2.63 \mathrm{ppm}$ were attributed to the methene hydrogen $\left(-\mathrm{COCH}_{2} \mathrm{CH}_{2}-\right)$ of the succinyl group of TS [26]. These spectra proved the formation of TS-GC. 


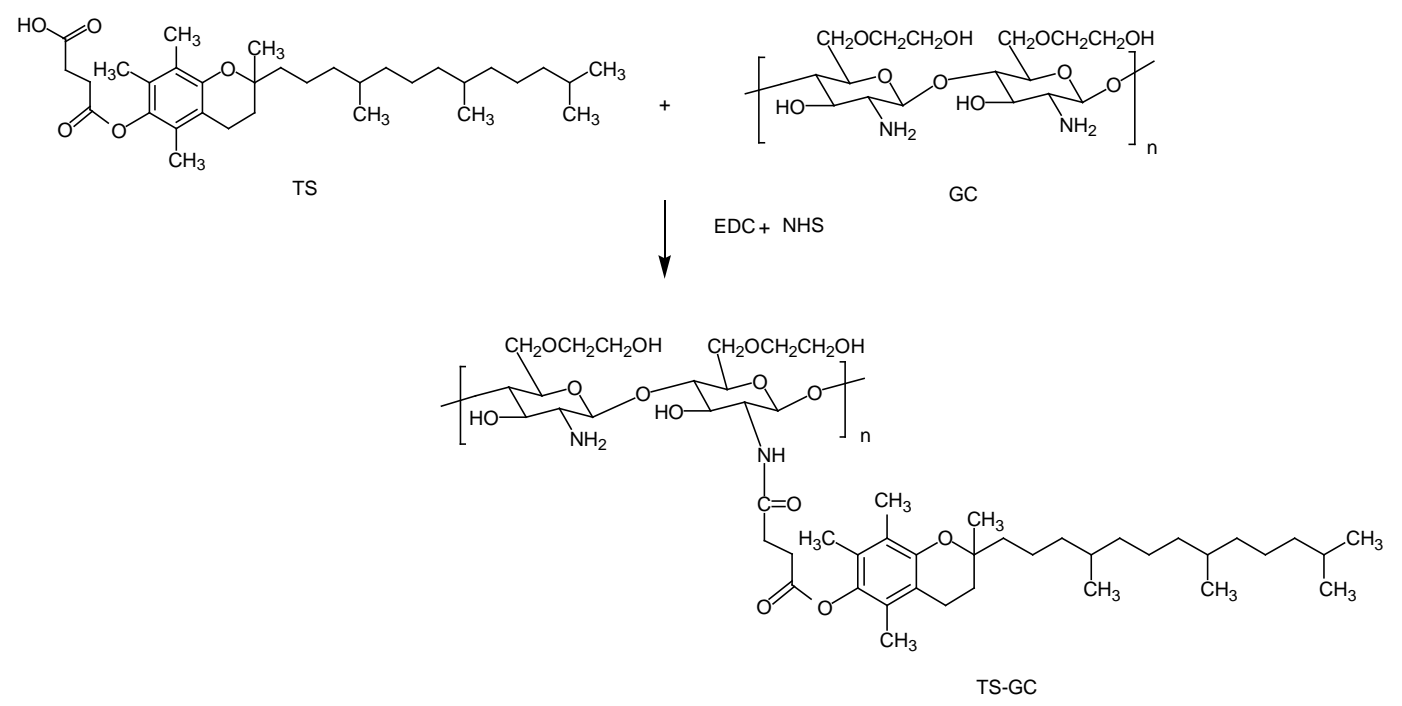

Figure 1. Synthesis of $\alpha$-tocopherol succinate modified glycol chitosan (TS-GC).
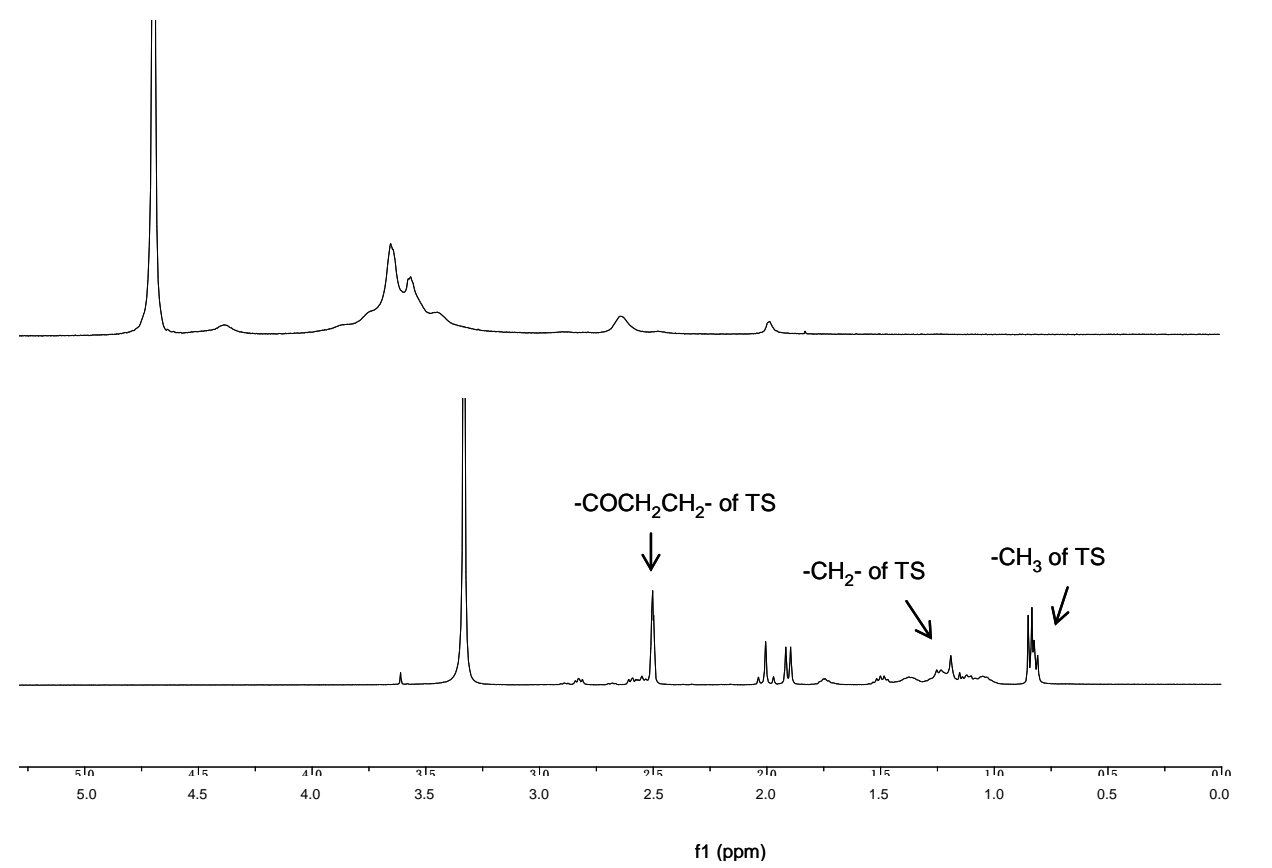

Figure 2. Proton nuclear magnetic resonance $\left({ }^{1} \mathrm{H}-\mathrm{NMR}\right)$ spectra of (a) glycol chitosan (GC) and (b) TS-GC.

FT-IR was used to characterize the functional groups of GC before and after modification. The FT-IR spectra of GC (a), TS-GC (b), physical mixture of GC and TS (c) and TS (d) are shown in Figure 3. In curve a, GC showed characteristic signals at $3133 \mathrm{~cm}^{-1}(\mathrm{O}-\mathrm{H}$ stretch overlapped with $\mathrm{N}-\mathrm{H}$ stretch), $1669 \mathrm{~cm}^{-1}$ (amide I band, C=O stretch of acetyl group), $1558 \mathrm{~cm}^{-1}$ (amide II band, $\mathrm{N}-\mathrm{H}$ bending) and $1399 \mathrm{~cm}^{-1}$ (C-H bending), respectively. The spectrum of TS (curve d) showed characteristic peaks of $\mathrm{C}=\mathrm{O}$ bond at $1754 \mathrm{~cm}^{-1}$ (the carbonyl of ester bond) and at $1715 \mathrm{~cm}^{-1}$ (carboxylic $\mathrm{C}=\mathrm{O}$ ). And the peak at $934 \mathrm{~cm}^{-1}$ was attributed to the out-of-plane bending vibrations of carboxylic $\mathrm{C}-\mathrm{OH}$. Compared with GC, the increased intensity of peaks at $1669 \mathrm{~cm}^{-1}$ and $1558 \mathrm{~cm}^{-1}$ in TS-GC (curve b) indicated the formation of amide bond. In addition, disappearance of carboxylic $\mathrm{C}=\mathrm{O}$ signal at $1715 \mathrm{~cm}^{-1}$ and carboxylic $\mathrm{C}-\mathrm{OH}$ signal at $934 \mathrm{~cm}^{-1}$ further confirmed full reaction of TS with GC. All the above indicated the successful introduction of TS. 


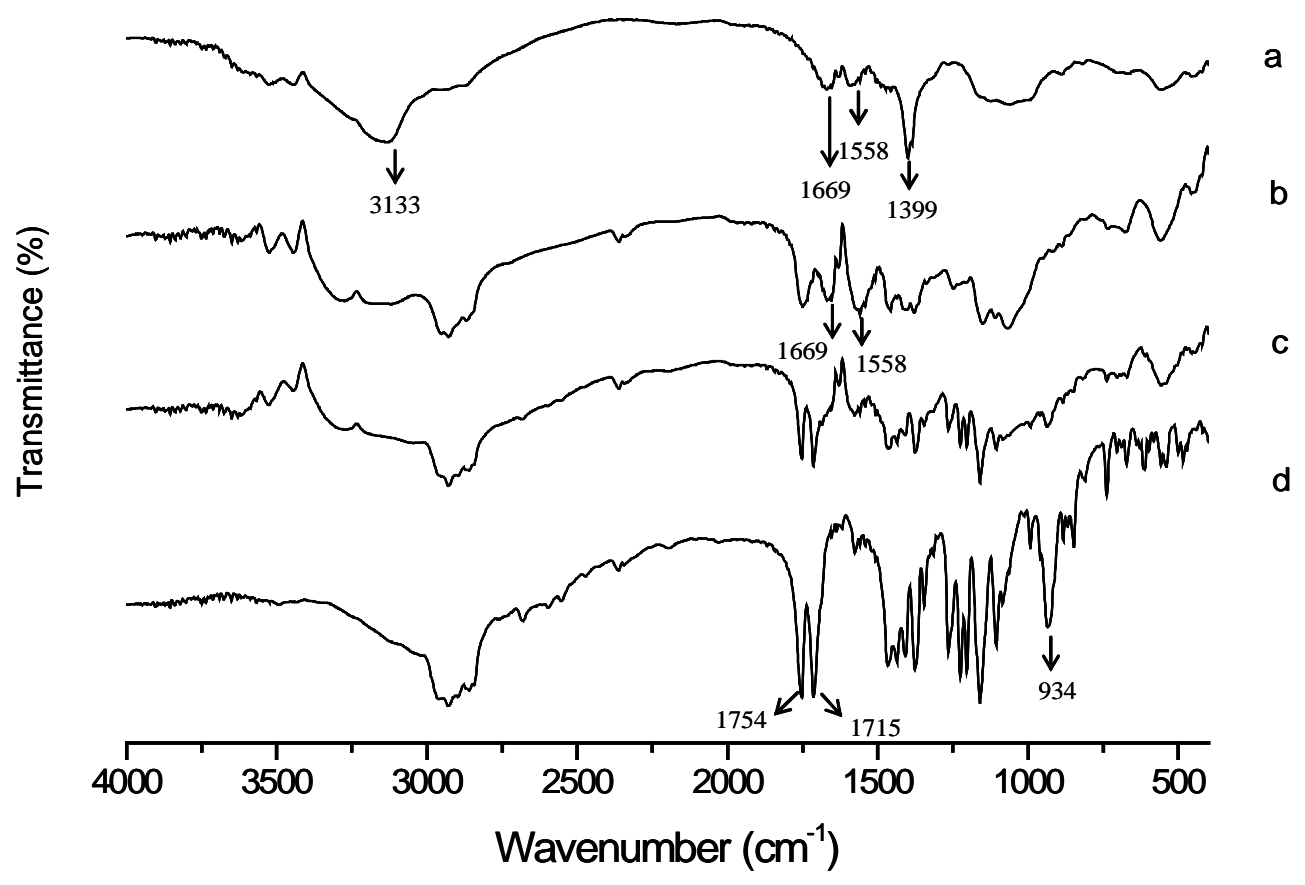

Figure 3. Fourier-transform infrared (FT-IR) spectra of (a) GC, (b) TS-GC, (c) physical mixture of GC and TS, and (d) TS.

The degree of amino substitution was calculated by measuring the amount of terminal amino groups of TS-GC with 2,4,6-trinitrobenzene sulphonic acid (TNBS reagent). The maximum absorbance of the yellow colour product was at $344 \mathrm{~nm}$, and the absorbance was in proportion to the number of primary amino groups. From the calibration curve obtained with unmodified GC solution with different concentrations, the substitution degree of TS-GC in this experiment was calculated as $11.3 \%$.

Critical aggregation concentration (CAC) plays an important role in maintaining the stability of micelles upon dilution. Only when the concentration of the polymer is higher than its CAC can micelles be formed. Polymeric micelles are generally more stable because of their markedly lower CAC. For the determination of the CAC of TS-GC, the pyrene fluorescence was employed to monitor the properties of TS-GC in solution. This method utilized pyrene's sensitivity to the local polarity of the environment. Below the $\mathrm{CAC}$, the polymers only exist as single chains, and pyrene is solubilized in water. When the concentration increases to reach the critical value called CAC, polymer chains start to associate to form micelles, and pyrene partitions preferentially toward the hydrophobic cores of micelles. This leads to the increase of fluorescence intensity, and the intensity of the third peak increased significantly compared to that of the first peak. So the CAC values were calculated from curves of $\mathrm{I}_{3} / \mathrm{I}_{1}$ versus polymer concentration. The $\mathrm{CAC}$ was defined as the intercept of the tangents to the curve before and after the point of inflection [27]. For TS-GC, the CAC was calculated to be $7.2 \times 10^{-3} \mathrm{mg} / \mathrm{mL}$, which was significantly lower than that of the low molecular weight surfactants in water. This implied the TS-GC micelles could be stable and not easily dissociate upon dilution.

\subsection{Preparation of TS-GC Micelles}

In general, the aggregates of amphiphilic polymers can be prepared through diafiltration or sonication methods. As for diafiltration, the polymer was dissolved in co-solvent and then dialyzed against water. For TS-GC, it was not easily dissolved in the mixture of organic solvent with water, so the probe-sonication was employed. Micelle formation is a delicate balance between the attractive force that leads to the association of molecules and the repulsive force that prevents unlimited growth of the micelles [28]. It was reported that once the micelle structure was formed completely, the drugs were hardly to be incorporated into the micelles [29]. So, in this study, in order to get high 
encapsulation efficiency, the PTX loading occurred simultaneously with self-assembly of TS-GC under the probe-sonication treatment as stated in Section 3.5. The PTX encapsulation efficiency reached to $71.8 \%$, and the drug loading capacity was calculated as $8.0 \%$.

\subsection{Characterization of PTX-Loaded TS-GC Micelles}

\subsubsection{XRD Analysis}

To confirm the existence form of PTX in PTX-loaded TS-GC micelles, XRD analysis was conducted for PTX, blank micelles, their physical mixture and PTX-loaded micelles. As illustrated in Figure 4, typical intense diffraction peaks of PTX were still observed with weak intensity in the pattern obtained from the physical mixture of PTX and blank micelles, which reflected the presence of PTX crystal in the mixture. While the spectrum of lyophilized PTX-loaded micelles was similar to that of the blank micelles, and there were no diffraction peaks for PTX. It can be concluded that PTX was encapsulated in the polymeric micelles in molecular or amorphous state and there was no free drug on the surface of micelles.

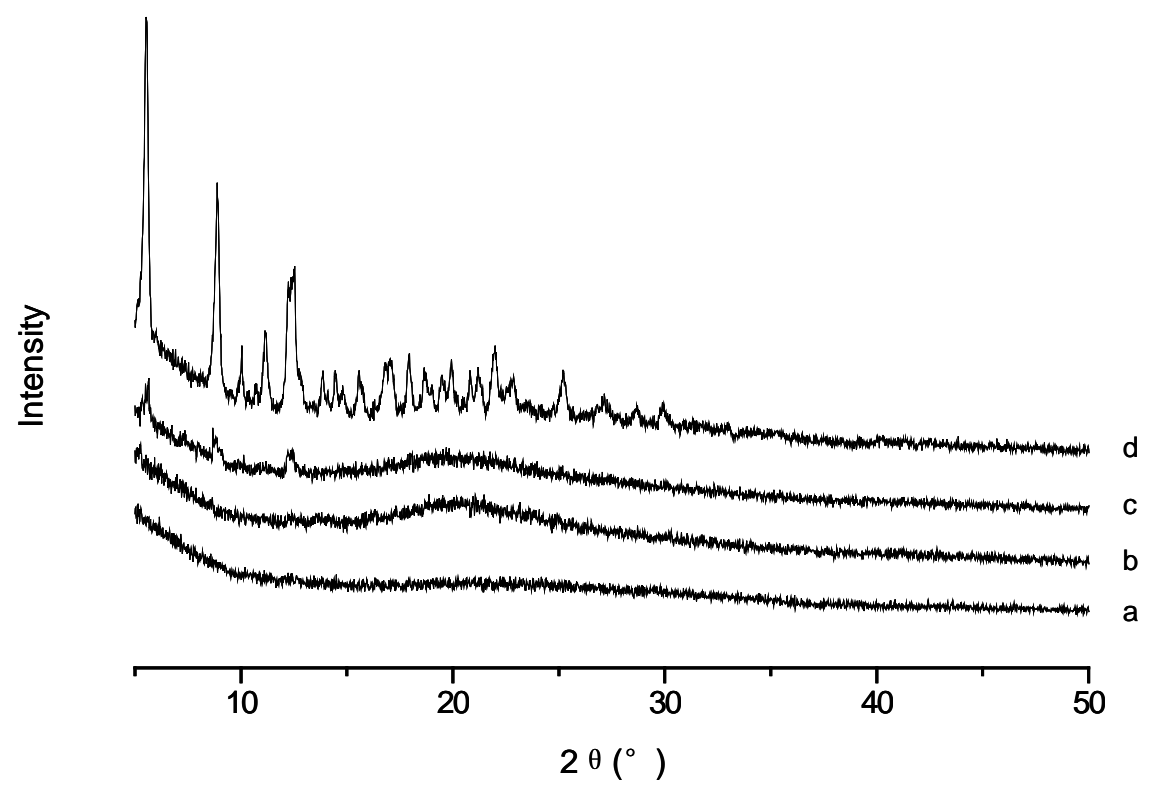

Figure 4. X-ray diffraction (XRD) spectra of (a) blank micelles; (b) paclitaxel (PTX)-loaded micelles; (c) physical mixture of PTX and blank micelles; and (d) PTX.

\subsubsection{Particle Size and Zeta Potential}

Size of the micelles is an important factor affecting the in vivo fate of the drug. In this study, the particle size and their distribution of the micelles were measured by dynamic light scattering method. From the result, the mean particle size of PTX-loaded micelles was $142 \mathrm{~nm}$ with polydispersity index (PDI) of 0.186, and it was larger than that of blank micelles ( $35 \mathrm{~nm}$, PDI of 0.105), which indicated the encapsulation of PTX into the micelles. For both bare and PTX-loaded micelles, the size distribution was narrow. It was reasonably safe to assume an increasing accumulation of the drug in tumor tissue. Because it was reported that the small size of micelles $(<200 \mathrm{~nm})$ can reduce non-selective clearance by the reticuloendothelial system (RES) and show EPR effect for passive accumulation in certain tumor sites [30]. Moreover, the size-sieving may occur during the distribution process in the body, and the narrow size distribution may promote the selective accumulation at the target site. Transmission electron microscope (TEM) micrograph of PTX-loaded micelles is shown in Figure 5. It was obvious that the micelles were spherical in shape. 


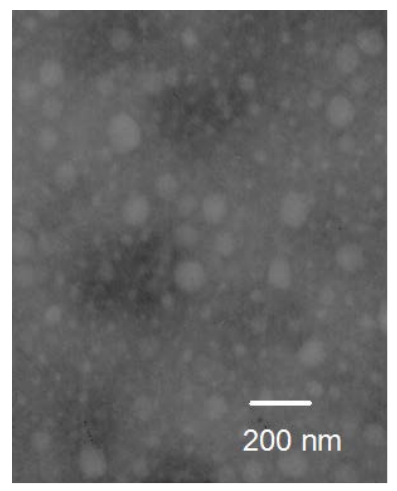

Figure 5. Transmission electron microscope (TEM) image of PTX-loaded micelles.

For a micellar solution, zeta potential can greatly influence the particle stability through the electrostatic repulsion. Relatively high surface charge could provide a repelling force between the particles, and thus increase the stability of the system. In this study, the blank micelles and PTX-loaded micelles in aqueous medium were positively charged with zeta potential of $+19 \mathrm{mV}$ and $+16 \mathrm{mV}$, respectively, due to the presence of ionized amino groups of TS-GC distributing on the surface of micelles. It is reasonable to confirm the aqueous stability of the micelles.

\subsection{In Vitro Hemolytic Test}

As analogs of low molecular weight surfactants, amphiphilic polymers may solubilize lipids or be inserted into phospholipid membranes, and consequently lead to hemolysis of red blood cells following intravenous administration [31]. It is necessary to determine whether TS-GC induces hemolysis and is safe for intravenous injection. The hemolysis of PTX-loaded TS-GC micelles was compared with that of Cremophor EL-based formulation. It was observed that in the range of $10-200 \mu \mathrm{g} / \mathrm{mL}$, the hemolysis of PTX-loaded TS-GC micelles was almost negligible, with only $4.2 \%$ at the concentration of $200 \mu \mathrm{g} / \mathrm{mL}$, while the hemolysis induced by the Cremophor EL-based formulation increased from $0.04 \%$ to $10.9 \%$. The results suggested that PTX-loaded micelles were not toxic to the erythrocytes.

\subsection{In Vitro Cytotoxicity Study}

The cytotoxicity study was conducted by 3-(4,5-dimethylthiazol-2-yl)-2,5-diphenyl tetrazolium bromide (MTT) method to assess the effectiveness of PTX-loaded TS-GC micelles. As shown in Figure 6, the cytotoxicity of PTX-loaded TS-GC micelles was similar to that of Cremophor EL-based formulation with equivalent doses in the concentration ranges used in this study. Furthermore, as the concentration and incubation time increased, PTX-loaded micelles and Cremophor EL-based formulation displayed increasing cytotoxicity. It was straightforward to understand that both concentration and incubation time played a major role in the in vitro cytotoxicity of PTX. For longer incubation periods, a larger number of cells enter the G2 and M cell cycle phases, during which PTX is more active. The findings suggested that PTX-loaded TS-GC micelles could be used as a potential PTX carrier. 


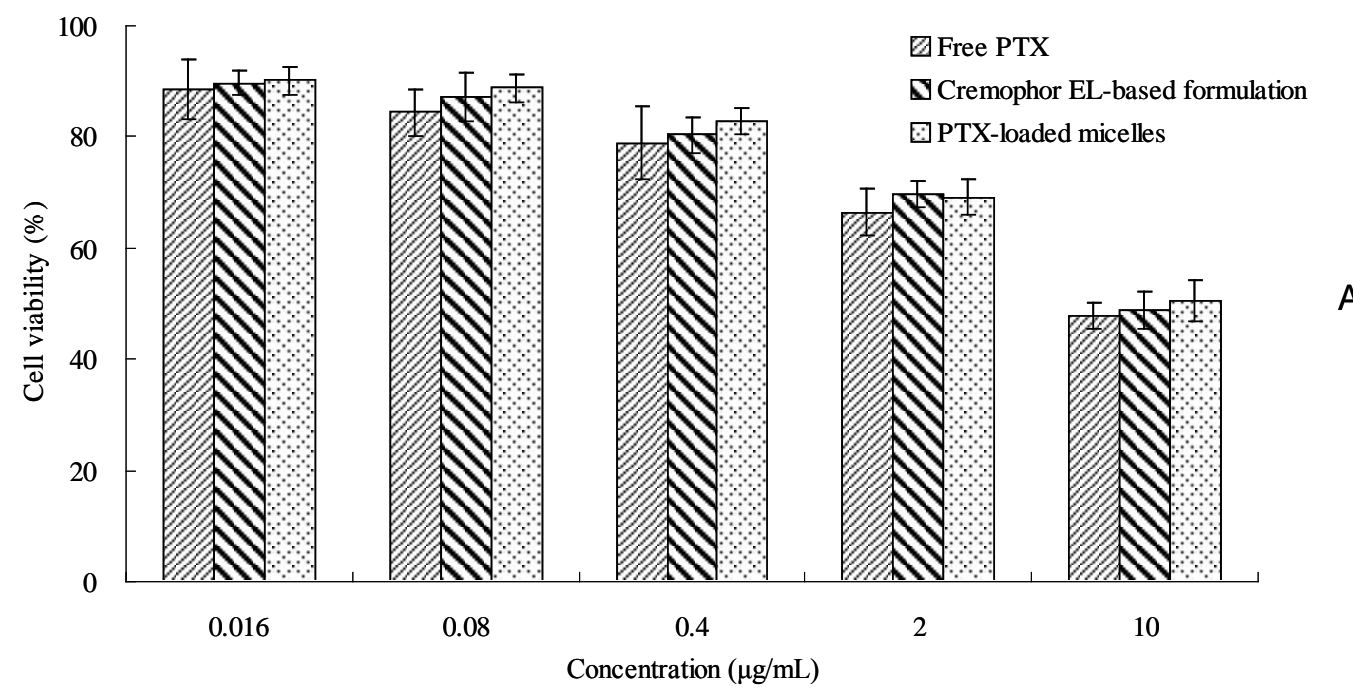

A

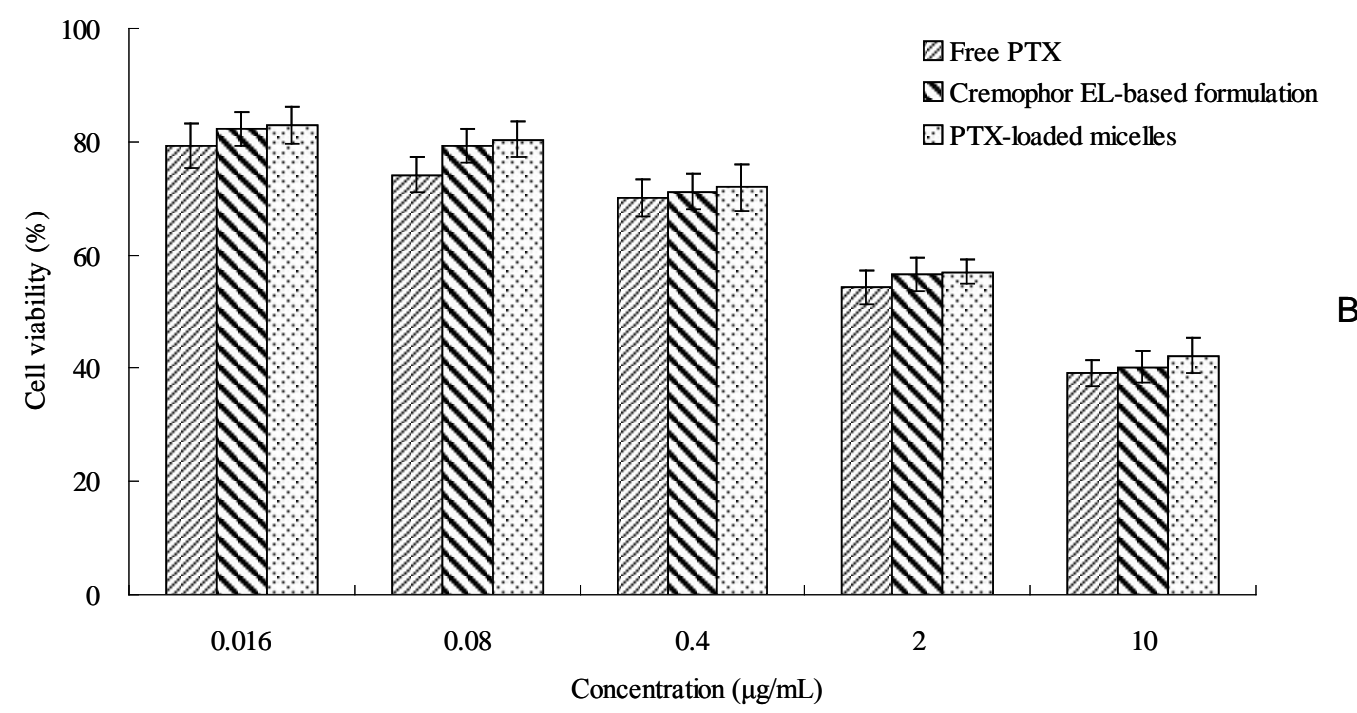

Figure 6. In vitro cytotoxicity of free PTX in dimethyl sulfoxide (DMSO), Cremophor EL-based formulation and PTX-loaded micelles against MCF-7 cells after $24 \mathrm{~h}$ (A) and $48 \mathrm{~h}$ (B) incubation (mean $\pm \mathrm{SD}, n=3$ ).

\subsection{In Vivo Antitumor Activity Study}

The antitumor efficacy of PTX-loaded micelles in vivo was consistent well with the in vitro cell experiment. As shown in Figure 7, comparing with the saline control, a striking antitumor response was observed in all the treatment groups $(p<0.05)$. Furthermore, PTX-loaded micelles and Cremophor EL-based formulation had the similar antitumor efficacy at the same dose of $10 \mathrm{mg} / \mathrm{kg}$, with tumor inhibition rate (TIR) of $72.3 \%$ and $69.6 \%$, respectively, and the difference between the two groups was not statistically significant. The slight superior of the micelles might be explained by the increased concentration of PTX in tumor tissue due to the EPR effect, and the exact mechanism will be further studied. 


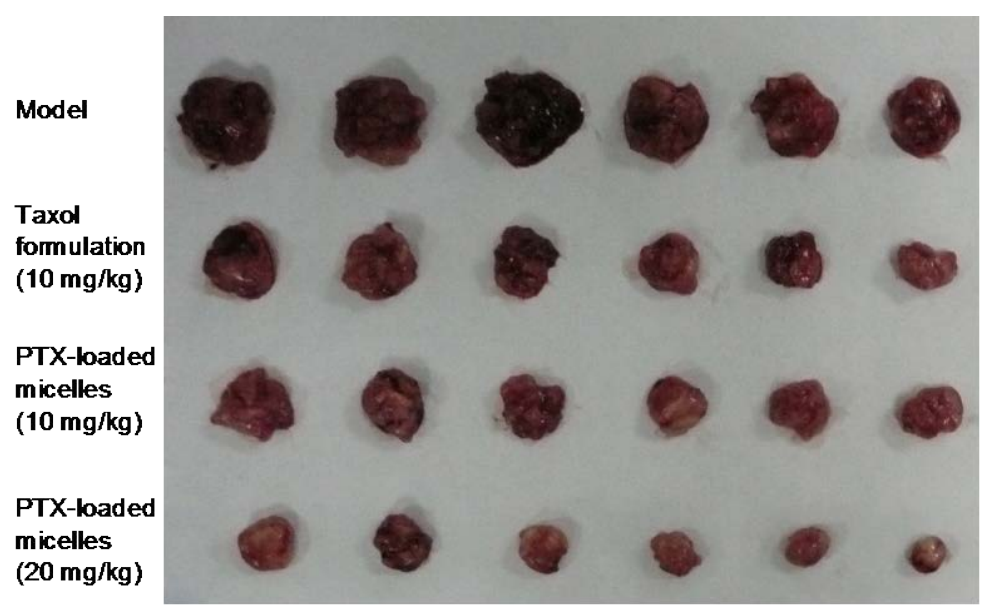

Figure 7. Photograph of tumors from each treatment group excised after intravenous injection treatment on day 13.

On the other hand, the toxic effect of PTX-loaded micelles was much less than that of the Cremophor EL-based formulation. At the high dose of $20 \mathrm{mg} / \mathrm{mL}$, the intravenous administration of Cremophor EL-based formulation resulted in development of immediate ataxia and enhanced respiration, and 3 in 6 animals died although the injection speed was slowed down and the first aid treatment was given immediately after the symptoms appeared. In contrast, none of the mice treated with PTX-loaded micelles died throughout the study. This may be explained by the serious side effect of Cremophor EL. While for the micelles, all the components constructed TS-GC were nontoxic and biocompatible. It was obvious that the low toxicity of PTX-loaded micelles is of great value.

Taken together, these results implied that PTX-loaded TS-GC micelles not only had similar to better antitumor efficacy as Cremophor EL-based formulation, but also induced less systemic toxicity, especially at the higher dose. The PTX-loaded micelles might allow the administration of a higher dose of PTX.

\section{Materials and Methods}

\subsection{Materials}

Glycol chitosan (Mw $250 \mathrm{kDa}$, deacetylation degree of 82.7\%), 2,4,6-trinitrobenzene sulfonic acid (TNBS), pyrene (purity $>99 \%$ ) and 3-(4,5-dimethylthiazol-2-yl)-2,5-diphenyl tetrazolium bromide (MTT) were purchased from Sigma, St. Louis, MO, USA. 1-Ethyl-3-(3-dimethylaminopropyl) carbodiimide hydrochloride (EDC) and $N$-hydroxysuccinimide (NHS) were obtained from Shanghai Medpep Co., Ltd., Shanghai, China. $\alpha$-Tocopherol succinate (TS) was kindly donated by Xinchang Pharmaceutical Co., Ltd., Shaoxing, China. Paclitaxel (PTX, purity of 99.9\%) was supplied by Tianfeng Bioengineering Technology Co., Ltd., Shenyang, China. Cremophor EL was a kind gift from BASF Corp., Ludwigshafen, Germany. Dulbecco's modified Eagle's medium (DMEM), fetal bovine serum (FBS), and penicillin-streptomycin mixture were purchased from Gibco BRL, Carlsbad, CA, USA. All other chemicals and solvents were of analytical or chromatographic grade and used without further purification. Distilled water or Milli-Q water was used in all experiments.

\subsection{Animals and Cell Line}

The male New Zealand rabbit (weighing $2 \mathrm{~kg}$ ) and specific pathogen-free female Kunming mice (5-6 weeks old, weighing 20-25 g) were acquired from Laboratory Animal Center of Harbin Medical University, Harbin, China. MCF-7 cells (human breast cancer cells) were obtained from American Type Culture Collection, Manassas, VA, USA. U14 cells (mouse uterine cervix carcinoma cells) were provided by the Cell Resource Center of Chinese Academy of Medical Sciences, Beijing, China. All 
animal procedures were performed in compliance with the animal care protocols approved by the Animal Ethics Committee of Harbin Medical University (18 May 2017, No. 201705180118).

\subsection{Synthesis of $\alpha$-Tocopherol Succinate Modified Glycol Chitosan (TS-GC)}

$\alpha$-Tocopherol succinate modified glycol chitosan (TS-GC) was synthesized by the coupling reaction between carboxyl group of TS with amine group of GC in the presence of EDC [32]. Briefly, GC (100 mg) was dissolved in $10 \mathrm{~mL}$ of distilled water and TS $(64 \mathrm{mg})$ was dissolved in $10 \mathrm{~mL}$ of methanol. The TS solution was slowly added to the GC solution and followed by the addition of excessive EDC and NHS under gentle stirring at room temperature. After overnight reaction, the mixture was dialyzed against distilled water using a cellulose membrane (Viskase, Willowbrook, IL, USA, MWCO: 7000), and TS-GC was obtained by freeze-drying of the dialyzed solution.

\subsection{Characterization of TS-GC}

To confirm the formation of TS-GC, proton nuclear magnetic resonance $\left({ }^{1} \mathrm{H}-\mathrm{NMR}\right)$ analysis was operated at $300 \mathrm{MHz}$ using Bruker Avance spectrometer (AV-300, Bruker, Karlsruhe, Germany). GC and the conjugate were dissolved in $\mathrm{D}_{2} \mathrm{O}$ and DMSO-d 6 at the concentration of $1 \%(w / v)$, respectively.

In order to further investigate the structural changes during the synthesis process, Fourier-transform infrared (FT-IR) spectra of GC, TS, their physical mixture and the conjugate were obtained on FT-IR spectrometer (Tensor II, Bruker, Fällanden, Switzerland) in the range between 4000 and $400 \mathrm{~cm}^{-1}$ after compressed into $\mathrm{KBr}$ pellets.

The degree of substitution means the number of tocopherol groups per 100 anhydroglucose units (amino groups) of TS-GC. The amount of remaining terminal amino residues on TS-GC was measured using 2,4,6-trinitrobenzene sulfonic acid (TNBS reagent) according to TNBS method [33,34]. The numerical value was calculated from the calibration curve that obtained by GC solution.

The critical aggregation concentration (CAC) of TS-GC in aqueous milieu was measured using a fluorometer (F-2500 FL Spectrophotometer, Hitachi Ltd., Tokyo, Japan). The pyrene fluorescence was monitored to evaluate the micropolarity and hydrophobicity of the region in which it was solubilized, so as to prove the formation of the micelles [35].

\subsection{Preparation of PTX-Loaded TS-GC Micelles}

PTX-loaded TS-GC micelles were prepared by a probe-type ultrasonic method. Briefly, $10 \mathrm{mg}$ of TS-GC was swollen in $10 \mathrm{~mL}$ of distilled water under gentle stirring overnight at room temperature to ensure complete dispersion. PTX solution was prepared by dissolving PTX in methanol at the concentration of $1 \mathrm{mg} / \mathrm{mL}$. The PTX solution was added into the TS-GC solution, and the final mixture was sonicated for $10 \mathrm{~min}$ at $400 \mathrm{~W}$ in an ice bath by a ultrasonicator (JY92-II, Ningbo Scientz Biotechnology Co., Ltd., Ningbo, China). The sonication was carried out with the pulse function (turned on for $3 \mathrm{~s}$ and off for $2 \mathrm{~s}$ ). The unloaded PTX was removed by centrifugation at $4000 \mathrm{rpm}$ for $10 \mathrm{~min}$. The resulting supernatant was lyophilized to obtain PTX-loaded TS-GC micelles. The blank micelles were prepared by the same procedure except no PTX was added.

\subsection{Characterization of PTX-Loaded TS-GC Micelles}

\subsubsection{X-ray Diffraction (XRD) Analysis}

X-ray diffraction diagrams were detected using an X-ray diffractometer (Geigerflex, Rigaku Co., Akishima, Japan) with $\mathrm{Cu} \mathrm{K} \alpha$ radiation. Samples were scanned from 5 to $50^{\circ}(2 \theta)$ at a scanning speed of $2^{\circ} / \mathrm{min}$ and step size of $0.02^{\circ}$. The $\mathrm{X}$-ray system was operated at a potential of $30 \mathrm{kV}$ and current of $30 \mathrm{~mA}$. 


\subsubsection{Transmission Electron Microscopy (TEM) Observation}

Morphology of the micelles was observed using a transmission electron microscope (TEM) (Jeol JEM1200EX, Tokyo, Japan) that operated at an accelerating voltage of $60 \mathrm{kV}$. For TEM, an aqueous droplet of micelles was immobilized on copper grids and negatively stained with phosphotungstate solution $(2 \%, w / v)$, then dried at room temperature before observation.

\subsubsection{Measurement of Particle Size and Zeta Potential}

The number-weighted diameter, their distribution, and zeta potential of the micelles were measured using photon correlation spectroscopy with a Zetasizer Nano-ZS90 at $25^{\circ} \mathrm{C}$. The lyophilized samples were suspended in distilled water before measurement.

\subsubsection{Determination of Drug Loading and Drug Encapsulation Efficiency}

The drug loading and entrapment efficiency of the PTX-loaded micelles were determined as follows: $100 \mu \mathrm{L}$ of PTX-loaded TS-GC micelles solution (concentration of TS-GC $=1.0 \mathrm{mg} / \mathrm{mL}$ ) was centrifuged at 10,000 rpm for $10 \mathrm{~min}$ with ultrafilter (Vivaspin 500, MWCO $10 \mathrm{k}$, Sartorius Co., Göttingen, Germany). The unentrapped PTX amount in the ultrafiltrate $\left(W_{1}\right)$ was determined using HPLC method. The HPLC system consisted of a mobile phase delivery pump (LC-10ATVP HPLC pump, Shimadzu, Kyoto, Japan) and a UV detector (SPD-10A UV/Vis detector, Shimadzu, Japan). For separation, a Diamonsil ${ }^{\mathrm{TM}} \mathrm{C}_{18}$ reverse-phase column $(200 \times 4.6 \mathrm{~mm}, 5 \mu \mathrm{m}$, Dikma technologies Inc., Beijing, China) was used. The mobile phase consisted of a mixture of acetonitrile and water (60:40, $v / v)$. The detector wavelength, column temperature, and flow rate of the mobile phase were set at $227 \mathrm{~nm}, 30^{\circ} \mathrm{C}$ and $1.0 \mathrm{~mL} / \mathrm{min}$, respectively. The injection volume of the test samples was $20 \mu \mathrm{L}$.

In order to measure the total PTX content $\left(W_{0}\right)$ in the micelles, another $100 \mu \mathrm{L}$ of identical PTX-loaded micelles solution was ultrasonicated in $10 \mathrm{~mL}$ methanol to extract PTX from the micelles. The encapsulation efficiency (EE\%) and drug loading (DL\%) of PTX were calculated using the following Equations (1) and (2):

$$
\begin{gathered}
\mathrm{EE} \%=\left(W_{0}-W_{1}\right) / W_{0} \times 100 \% \\
\mathrm{DL} \%=\left(W_{0}-W_{1}\right) /\left(W_{0}-W_{1}+100\right) \times 100 \%
\end{gathered}
$$

where $W_{1}$ is the PTX content in the ultrafiltrate, and $W_{0}$ is the total PTX amount in the solution. The unit was microgram.

\subsection{In Vitro Hemolysis Test}

Hemolysis assessment of the TS-GC micelles was conducted as the method reported by Gong et al. [36]. The rabbit blood, freshly drawn from the ear vein, was centrifuged at $3000 \mathrm{rpm}$ for $10 \mathrm{~min}$ to isolate the erythrocytes. The erythrocytes were washed with normal saline for three times to make the supernatant achromatic, and then suspended in normal saline to get a $2 \%(v / v)$ suspension. The lyophilized powder of PTX-loaded TS-GC micelles was dispersed in $0.9 \% \mathrm{NaCl}$, and different amounts of micelle solution were added into the tubes with $2.5 \mathrm{~mL}$ of $2 \%$ erythrocyte dispersion in each. Then adequate amounts of normal saline were added in every tube to obtain a final volume of $5 \mathrm{~mL}$. After incubating at $37^{\circ} \mathrm{C}$ for $4 \mathrm{~h}$, the mixture was centrifuged at $3000 \mathrm{rpm}$ for $10 \mathrm{~min}$ to remove intact red blood cells (RBC). The supernatant was analyzed for released hemoglobin at $540 \mathrm{~nm}$ using a spectrophotometer (UV-Vis Spectrophotometer Model 752, Shanghai Spectrum Instruments Co., Ltd., Shanghai, China). To obtain 0 and $100 \%$ hemolysis, $2.5 \mathrm{~mL}$ of saline and $2.5 \mathrm{~mL}$ of distilled water was added to $2.5 \mathrm{~mL}$ of RBC suspension, respectively. The degree of hemolysis was calculated by the following Equation (3).

$$
\text { Hemolysis }(\%)=\left(A_{\text {sample }}-A_{0 \%}\right) /\left(A_{100 \%}-A_{0 \%}\right) \times 100 \%
$$


where $A_{\text {sample }}, A_{0 \%}$, and $A_{100 \%}$ are the absorbance of the samples, a solution of $0 \%$ hemolysis, and a solution of $100 \%$ hemolysis, respectively.

\subsection{In Vitro Cytotoxicity}

The in vitro cytotoxic activity of samples was evaluated by MTT method using MCF-7 cell line [37]. Briefly, $50 \mu \mathrm{L}$ of MCF-7 cells growing in the logarithmic phase were cultured in Dulbecco's modified Eagle's medium (DMEM) containing 10\% $(v / v)$ fetal bovine serum, $100 \mathrm{IU} / \mathrm{mL}$ of penicillin $\mathrm{G}$ sodium and $100 \mu \mathrm{g} / \mathrm{mL}$ of streptomycin sulfate. The cells were seeded in a 96-well microtitre plate at the density of $1 \times 10^{4}$ cells per well and maintained in an incubator supplied with $5 \% \mathrm{CO}_{2}$ at $37{ }^{\circ} \mathrm{C}$. After reaching $75 \%$ confluence, the cells were incubated with free drug in dimethyl sulfoxide (DMSO), Cremophor EL-based PTX formulation and PTX-loaded TS-GC micelles at the equivalent drug concentrations ranging from 0.016 to $10 \mu \mathrm{g} / \mathrm{mL}$ for 24 and $48 \mathrm{~h}$. At designated time intervals, the medium was removed and the wells were washed with PBS for two times. Then, $10 \mu \mathrm{L}$ of MTT $(5 \mathrm{mg} / \mathrm{mL}$ in PBS) was added to the wells. After an additional $4 \mathrm{~h}$ of incubation, the MTT medium was aspirated off and $100 \mu \mathrm{L}$ of DMSO was added to each well to dissolve the formazan crystals. The absorbance was measured at $570 \mathrm{~nm}$ with a microplate reader (Bio-Tek Instruments Inc., Vernusky, VT, USA). Untreated cells were taken as the control with $100 \%$ viability, and cells without addition of MTT were used as blank to calibrate the spectrophotometer to zero absorbance. The cytotoxicity was calculated as follows Equation (4):

$$
\text { Cytotoxicity }=\left(\mathrm{A}_{\text {culture medium }}-\mathrm{A}_{\text {sample }}\right) / \mathrm{A}_{\text {culture medium }} \times 100 \%
$$

where $\mathrm{A}_{\text {culture medium }}$ and $\mathrm{A}_{\text {sample }}$ are the absorbance of cells incubated with culture medium and absorbance of cells exposed to the sample, respectively.

\subsection{In Vivo Antitumor Activity}

Specific pathogen-free Kunming mice, 5 to 6 weeks old, weighing 20-25 g were used for this study. The animals were housed six per cage in standard size cages under standard laboratory conditions ( $21 \pm 2{ }^{\circ} \mathrm{C}, 12$-h light: 12 -h dark cycle, relative humidity of 50-60\%) and allowed to access sterilized food and water freely. The mice were left to acclimatize for a week prior to the experiment.

U14 cells of the third passage in vivo were used for tumor development. Animals were inoculated with $2.0 \times 10^{6}$ cells $(0.2 \mathrm{~mL} /$ mouse) subcutaneously in the armpit of right anterior limb. Three days later, the tumors were palpable, and the tumor model was established. The animals were randomized and divided into different groups $(n=6)$ : (1) normal saline group (negative control); (2) Cremophor EL-based PTX formulation groups (positive control, $10 \mathrm{mg} / \mathrm{kg}$ and $20 \mathrm{mg} / \mathrm{kg}$ ); and (3) PTX-loaded TS-GC micelles groups ( $10 \mathrm{mg} / \mathrm{kg}$ and $20 \mathrm{mg} / \mathrm{kg}$ ), and the treatments were initiated. All samples were injected intravenously via the tail vein every 3 days, four times in total. The day that mice received treatment was set as day 1 . At the end of the experiment, on day 13, the animals were sacrificed by cervical dislocation, and the tumors were excised, weighed and imaged. The tumor inhibition rate (TIR) of each formulation was defined as follows Equation (5):

$$
\begin{gathered}
\mathrm{TIR}=(\text { tumor weight of negative control group }- \text { tumor weight of treatment } \\
\text { group }) / \text { tumorweightofnegativecontrolgroup } \times 100 \%
\end{gathered}
$$

\subsection{Statistical Analysis}

Each experiment was performed in triplicate. Values were expressed as mean \pm standard deviation (SD). Statistical data analysis was performed using the Student's $t$-test with $p<0.05$ as the level of significance. 


\section{Conclusions}

In this study, a novel amphiphilic derivative of glycol chitosan was successfully synthesized by grafting $\alpha$-tocopherol succinate onto the skeleton of glycol chitosan. In aqueous milieu, the conjugates provide stable self-aggregates above the CAC. Furthermore, the water-insoluble anticancer agent, PTX, was successfully encapsulated into the core of the micelles. The mean diameters of PTX-loaded micelles were about $142 \mathrm{~nm}$, which was larger than blank ones. The spherical morphology of the micelles was visually confirmed by TEM. Moreover, the PTX-loaded TS-GC micelles possessed antitumor activities in vitro and in vivo, and had advantages over the commercially available Cremophor EL-based formulation in terms of low toxicity levels and increased tolerated dose. Therefore, this novel TS-GC polymer might be used as a potential carrier for PTX.

Author Contributions: N.L. and S.S. conceived and designed the experiments. N.L. performed the experiments, analyzed the data and wrote the paper; X.G. and Q.L. performed studies of in vitro cytotoxicity. F.C. and P.Y. gave us much useful advice and some pieces of guidance.

Funding: This work was funded by the National Natural Science Foundation of China (No. 51403057), Harbin Science and Technology Innovation Talents Special Fund Project (No. 2016RQQXJ097, No. 2016RQQXJ131), and the Doctoral Scientific Research Startup Foundation of Harbin Normal University (No. XKB201304).

Conflicts of Interest: The authors declare no conflict of interest.

\section{References}

1. Abou-ElNaga, A.; Mutawa, G.; El-Sherbiny, I.; Abd-ElGhaffar, H.; Allam, A.; Ajarem, J.; Mousa, S. Novel nano-therapeutic approach actively targets human ovarian cancer stem cells after xenograft into nude mice. Int. J. Mol. Sci. 2017, 18, 813. [CrossRef] [PubMed]

2. Bernabeu, E.; Cagel, M.; Lagomarsino, E.; Moretton, M.; Chiappetta, D.A. Paclitaxel: What has been done and the challenges remain ahead. Int. J. Pharm. 2017, 526, 474-495. [CrossRef] [PubMed]

3. Gelderblom, H.; Verweij, J.; Nooter, K.; Sparreboom, A. Cremophor EL: The drawbacks and advantages of vehicle selection for drug formulation. Eur. J. Cancer 2001, 37, 1590-1598. [CrossRef]

4. Liu, Y.; Zhang, B.; Yan, B. Enabling anticancer therapeutics by nanoparticle carriers: The delivery of paclitaxel. Int. J. Mol. Sci. 2011, 12, 4395-4413. [CrossRef] [PubMed]

5. Ravar, F.; Saadat, E.; Gholami, M.; Dehghankelishadi, P.; Mahdavi, M.; Azami, S.; Dorkoosh, F.A. Hyaluronic acid-coated liposomes for targeted delivery of paclitaxel, in-vitro characterization and in-vivo evaluation. J. Control. Release 2016, 229, 10-22. [CrossRef] [PubMed]

6. Hou, J.; Sun, E.; Zhang, Z.H.; Wang, J.; Yang, L.; Cui, L.; Ke, Z.C.; Tan, X.B.; Jia, X.B.; Lv, H.X. Improved oral absorption and anti-lung cancer activity of paclitaxel-loaded mixed micelles. Drug Deliv. 2017, 24, $261-269$. [CrossRef] [PubMed]

7. Alani, A.W.G.; Bae, Y.; Rao, D.A.; Kwon, G.S. Polymeric micelles for the pH-dependent controlled, continuous low dose release of paclitaxel. Biomaterials 2010, 31, 1765-1772. [CrossRef] [PubMed]

8. Erdoğar, N.; Esendağlı, G.; Nielsen, T.T.; Esendağl1-Yılmaz, G.; Yöyen-Ermiş, D.; Erdoğdu, B.; Sargon, M.F.; Eroğlu, H.; Bilensoy, E. Therapeutic efficacy of folate receptor-targeted amphiphilic cyclodextrin nanoparticles as a novel vehicle for paclitaxel delivery in breast cancer. J. Drug Target 2018, 26, 66-74. [CrossRef] [PubMed]

9. Nakamura, I.; Ichimura, E.; Goda, R.; Hayashi, H.; Mashiba, H.; Nagai, D.; Yokoyama, H.; Onda, T.; Masuda, A. An in vivo mechanism for the reduced peripheral neurotoxicity of NK105: A paclitaxel-incorporating polymeric micellar nanoparticle formulation. Int. J. Nanomed. 2017, 12, 1293-1304. [CrossRef] [PubMed]

10. Zhang, T.; Luo, J.; Fu, Y.; Li, H.; Ding, R.; Gong, T.; Zhang, Z. Novel oral administrated paclitaxel micelles with enhanced bioavailability and antitumor efficacy for resistant breast cancer. Colloids Surf. B Biointerfaces 2017, 150, 89-97. [CrossRef] [PubMed]

11. Torchilin, V.P. Micellar nanocarriers: Pharmaceutical perspectives. Pharm. Res. 2006, 24, 1-16. [CrossRef] [PubMed] 
12. Deshmukh, A.S.; Chauhan, P.N.; Noolvi, M.N.; Chaturvedi, K.; Ganguly, K.; Shukla, S.S.; Nadagouda, M.N.; Aminabhavi, T.M. Polymeric micelles: Basic research to clinical practice. Int. J. Pharm. 2017, 532, 249-268. [CrossRef] [PubMed]

13. Rapoport, N. Physical stimuli-responsive polymeric micelles for anti-cancer drug delivery. Prog. Polym. Sci. 2007, 32, 962-990. [CrossRef]

14. Biswas, S.; Kumari, P.; Lakhani, P.M.; Ghosh, B. Recent advances in polymeric micelles for anti-cancer drug delivery. Eur. J. Pharm. Sci. 2016, 83, 184-202. [CrossRef] [PubMed]

15. Kwon, G.S.; Okano, T. Polymeric micelles as new drug carriers. Adv. Drug Deliv. Rev. 1996, 21, $107-116$. [CrossRef]

16. Yang, Y.; Wang, S.; Wang, Y.; Wang, X.; Wang, Q.; Chen, M. Advances in self-assembled chitosan nanomaterials for drug delivery. Biotechnol. Adv. 2014, 32, 1301-1316. [CrossRef] [PubMed]

17. Prabaharan, M. Chitosan-based nanoparticles for tumor-targeted drug delivery. Int. J. Biol. Macromol. 2015, 72, 1313-1322. [CrossRef] [PubMed]

18. Fu, Y.-N.; Li, Y.; Li, G.; Yang, L.; Yuan, Q.; Tao, L.; Wang, X. Adaptive chitosan hollow microspheres as efficient drug carrier. Biomacromolecules 2017, 18, 2195-2204. [CrossRef] [PubMed]

19. Yu, J.; Liu, Y.; Zhang, L.; Zhao, J.; Ren, J.; Zhang, L.; Jin, Y. Self-aggregated nanoparticles of linoleic acid-modified glycol chitosan conjugate as delivery vehicles for paclitaxel: Preparation, characterization and evaluation. J. Biomater. Sci. Polym. Ed. 2015, 26, 1475-1489. [CrossRef] [PubMed]

20. Sharma, S.; Verma, A.; Teja, B.V.; Shukla, P.; Mishra, P.R. Development of stabilized paclitaxel nanocrystals: In-vitro and in-vivo efficacy studies. Eur. J. Pharm. Sci. 2015, 69, 51-60. [CrossRef] [PubMed]

21. Zhao, L.; Zhu, L.; Liu, F.; Liu, C.; Shan, D.; Wang, Q.; Zhang, C.; Li, J.; Liu, J.; Qu, X.; et al. pH triggered injectable amphiphilic hydrogel containing doxorubicin and paclitaxel. Int. J. Pharm. 2011, 410, 83-91. [CrossRef] [PubMed]

22. Park, J.S.; Han, T.H.; Lee, K.Y.; Han, S.S.; Hwang, J.J.; Moon, D.H.; Kim, S.Y.; Cho, Y.W. N-acetyl histidine-conjugated glycol chitosan self-assembled nanoparticles for intracytoplasmic delivery of drugs: Endocytosis, exocytosis and drug release. J. Control. Release 2006, 115, 37-45. [CrossRef] [PubMed]

23. Huo, M.; Fu, Y.; Liu, Y.; Chen, Q.; Mu, Y.; Zhou, J.; Li, L.; Xu, W.; Yin, T. N-mercapto acetyl- $N^{\prime}$-octyl-O, $N^{\prime \prime}$-glycol chitosan as an efficiency oral delivery system of paclitaxel. Carbohyd. Polym. 2018, 181, 477-488. [CrossRef] [PubMed]

24. Nielsen, P.B.; Müllertz, A.; Norling, T.; Kristensen, H.G. The effect of $\alpha$-tocopherol on the in vitro solubilisation of lipophilic drugs. Int. J. Pharm. 2001, 222, 217-224. [CrossRef]

25. Park, C.; Vo, C.L.-N.; Kang, T.; Oh, E.; Lee, B.-J. New method and characterization of self-assembled gelatin-oleic nanoparticles using a desolvation method via carbodiimide/ $N$-hydroxysuccinimide (EDC/NHS) reaction. Eur. J. Pharm. Biopharm. 2015, 89, 365-373. [CrossRef] [PubMed]

26. Xu, X.; Li, L.; Zhou, J.; Lu, S.; Yang, J.; Yin, X.; Ren, J. Preparation and characterization of $N$-succinyl- $N^{\prime}$-octyl chitosan micelles as doxorubicin carriers for effective anti-tumor activity. Colloids Surf. B Biointerfaces 2007, 55, 222-228.

27. Băran, A.; Stîngă, G.; Anghel, D.F.; Iovescu, A.; Tudose, M. Comparing the spectral properties of pyrene as free molecule, label and derivative in some colloidal systems. Sens. Actuators B Chem. 2014, 197, 193-199. [CrossRef]

28. Astafieva, I.; Zhong, X.F.; Eisenberg, A. Critical micellization phenomena in block polyelectrolyte solutions. Macromolecules 1993, 26, 7339-7352. [CrossRef]

29. Zhang, C.; Ping, Q.; Zhang, H. Self-assembly and characterization of paclitaxel-loaded N-octyl-O-sulfate chitosan micellar system. Colloids Surf. B Biointerfaces 2004, 39, 69-75. [CrossRef] [PubMed]

30. Danhier, F.; Danhier, P.; De Saedeleer, C.J.; Fruytier, A.-C.; Schleich, N.; Rieux, A.d.; Sonveaux, P.; Gallez, B.; Préat, V. Paclitaxel-loaded micelles enhance transvascular permeability and retention of nanomedicines in tumors. Int. J. Pharm. 2015, 479, 399-407. [CrossRef] [PubMed]

31. Huo, M.; Zhang, Y.; Zhou, J.; Zou, A.; Yu, D.; Wu, Y.; Li, J.; Li, H. Synthesis and characterization of low-toxic amphiphilic chitosan derivatives and their application as micelle carrier for antitumor drug. Int. J. Pharm. 2010, 394, 162-173. [CrossRef] [PubMed]

32. Liang, N.; Sun, S.; Li, X.; Piao, H.; Piao, H.; Cui, F.; Fang, L. $\alpha$-Tocopherol succinate-modified chitosan as a micellar delivery system for paclitaxel: Preparation, characterization and in vitro/in vivo evaluations. Int. J. Pharm. 2012, 423, 480-488. [CrossRef] [PubMed] 
33. Bernkop-Schnürch, A.; Krajicek, M.E. Mucoadhesive polymers as platforms for peroral peptide delivery and absorption: Synthesis and evaluation of different chitosan-EDTA conjugates. J. Control. Release 1998, 50, 215-223. [CrossRef]

34. Dosio, F.; Brusa, P.; Crosasso, P.; Arpicco, S.; Cattel, L. Preparation, characterization and properties in vitro and in vivo of a paclitaxel-albumin conjugate. J. Control. Release 1997, 47, 293-304. [CrossRef]

35. Yan, M.; Li, B.; Zhao, X. Determination of critical aggregation concentration and aggregation number of acid-soluble collagen from walleye pollock (Theragra chalcogramma) skin using the fluorescence probe pyrene. Food Chem. 2010, 122, 1333-1337. [CrossRef]

36. Gong, J.; Huo, M.; Zhou, J.; Zhang, Y.; Peng, X.; Yu, D.; Zhang, H.; Li, J. Synthesis, characterization, drug-loading capacity and safety of novel octyl modified serum albumin micelles. Int. J. Pharm. 2009, 376, 161-168. [CrossRef] [PubMed]

37. Laskar, P.; Samanta, S.; Ghosh, S.K.; Dey, J. In vitro evaluation of pH-sensitive cholesterol-containing stable polymeric micelles for delivery of camptothecin. J. Colloid Interface Sci. 2014, 430, 305-314. [CrossRef] [PubMed]

(C) 2018 by the authors. Licensee MDPI, Basel, Switzerland. This article is an open access article distributed under the terms and conditions of the Creative Commons Attribution (CC BY) license (http:/ / creativecommons.org/licenses/by/4.0/). 